LETTER TO THE EDITOR

\title{
Field-angle Dependence of the Zero-Energy Density of States in the Unconventional Heavy-Fermion Superconductor $\mathrm{CeCoIn}_{5}$
}

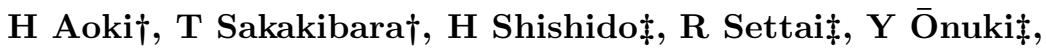 \\ $\mathbf{P}$ Miranović§ and $\mathrm{K}$ Machida§ \\ $\dagger$ Institute for Solid State Physics, University of Tokyo, Kashiwa, Chiba \\ 270-8581, Japan \\ ‡ Graduate School of Science, Osaka University, Toyonaka, Osaka 560-0043, \\ Japan \\ $\S$ Department of Physics, Okayama University, Okayama 700-8530, Japan
}

\begin{abstract}
Field-angle dependent specific heat measurement has been done on the heavy-fermion superconductor $\mathrm{CeCoIn}_{5}$ down to $\sim 0.29 \mathrm{~K}$, in a magnetic field rotating in the tetragonal $c$-plane. A clear fourfold angular oscillation is observed in the specific heat with the minima (maxima) occurring along the [100] ([110]) directions. Oscillation persists down to low fields $H \ll H_{c 2}$, thus directly proving the existence of gap nodes. The results indicate that the superconducting gap symmetry is most probably of $d_{x y}$ type.
\end{abstract}

Submitted to: J. Phys.: Condens. Matter

PACS numbers: 74.20.Rp,74.70.Tx,74.25.Bt,74.25.Op

E-mail: haoki@issp.u-tokyo.ac.jp 
Recently a new class of heavy-fermion superconductors $\mathrm{Ce} \operatorname{In}_{5}(T=\mathrm{Rh}, \mathrm{Ir}$, and Co) has been discovered. While $\mathrm{CeRhIn}_{5}[1$ is a pressure-induced superconductor, $\mathrm{CeIrIn}_{5}[2]$ and $\mathrm{CeCoIn}_{5}[3$ show superconductivity at an ambient pressure at 0.4 and $2.3 \mathrm{~K}$, respectively. Among these, unique properties of $\mathrm{CeCoIn}_{5}$ have attracted much attentions in these years. Various experiments such as specific heat [4, 5], thermal conductivity [4] and NMR $T_{1}$ measurements [6] have revealed that $\mathrm{CeCoIn}_{5}$ is an unconventional superconductor with line nodes in the gap. Together with the suppression of spin susceptibility below $T_{c}$ [6] [7, this compound is identified as a $d$ wave superconductor. Recent rf penetration depth measurement [8] and the flux line lattice imaging study by small-angle neutron scattering [9] also seem to be consistent with the existence of line nodes running along the $c$-axis.

In unconventional superconductors, identification of the gap-node structure is of fundamental importance in understanding the pairing mechanism. Regarding this issue, it has recently been pointed out that the zero-energy density of states (ZEDOS) in the superconducting mixed state exhibits a characteristic oscillation with respect to the angle between $H$ and the nodal direction [10 11, 12. An intuitive explanation of this effect employs a Doppler shift of the quasiparticle (QP) energy spectrum due to the local supercurrent flow 10, 11, 12, 13. More quantitative analysis of the effect has been done recently by Miranović et al [14] within the quasiclassical formalism, which incorporates also the contribution of QPs in the core states of vortices. Experimentally, the angular dependent ZEDOS can be probed by the thermal conductivity [15, 16, 17] or the specific heat [18] measurements in rotating magnetic field at low $T$. As for $\mathrm{CeCoIn}_{5}$, the angle-resolved thermal conductivity $(\kappa(\theta))$ measurement [17 has revealed a clear fourfold oscillation in a magnetic field rotating within the $c$-plane. It was argued that the line nodes exist along the [110] directions $\left(d_{x^{2}-y^{2}}\right.$ type gap symmetry).

The interpretation of the thermal conductivity data is, however, necessarily involved because $\kappa(\theta)$ is proportional to the specific heat $C(\theta)$ as well as to the QP scattering time $\tau(\theta)$. The latter also depends on the field orientation but with opposite angular oscillation amplitude to the former. Which of these two predominantly contributes to $\kappa(\theta)$ is a subtle question, making it difficult to identify the gap node direction in some cases. It is therefore of vital importance to directly observe ZEDOS by the angle-resolved specific heat measurement. In this Letter, we have examined the $C(H, \theta)$ of $\mathrm{CeCoIn}_{5}$ in a magnetic field rotating within the $c$-plane. We observed a clear fourfold oscillation in $C(H, \theta)$, and argue that the oscillation originates from the nodal gap most probably of $d_{x y}$ symmetry.

A single crystal of $\mathrm{CeCoIn}_{5}$ was grown by the so-called self-flux method [19]. The sample was cut into a thin plate $\left(\sim 2.3 \times 2.0 \times 0.7 \mathrm{~mm}^{3}\right)$ with the largest plane oriented perpendicular to the $c$-axis. Field-angle-dependent specific heat measurement was done by a standard adiabatic heat-pulse technique using a ${ }^{3} \mathrm{He}$ refrigerator. A transverse magnetic field was generated by a split-pair superconducting magnet and applied parallel to the $c$-plane of the sample mounted on a quartz platform. The ${ }^{3} \mathrm{He}$ refrigerator was mounted on a mechanical rotating stage driven by a computer controlled stepping motor at the top of the Dewar, by which a quasi-continuous change of the field direction with the minimum step of $0.04^{\circ}$ could be made. The angular dependent specific heat data were collected at an interval of $2^{\circ}$ in the range $\pm 105^{\circ}$ with respect to the $a$-axis. At the temperature of $380 \mathrm{mK}$, the $2^{\circ}$-rotation of the refrigerator caused a heating of the sample by $\sim 100 \mathrm{mK}$ in a field of $5 \mathrm{~T}$. In order to accurately evaluate $C(H, \theta)$ at constant temperature, each data point was taken 
after the sample temperature relaxed to within $0.1 \%$ of the initial temperature. The details of the experimental set-up will be published elsewhere 20. Field dependence of the specific heat up to $12.5 \mathrm{~T}$ was also measured by the relaxation method using a commercial calorimeter (PPMS, Quantum Design Co.).

Figure 1 shows the field dependence of the specific heat $C(H)$ of $\mathrm{CeCoIn}_{5}$ obtained at $T=0.38 \mathrm{~K}$ for $\boldsymbol{H} \|[100]$ and [110]. Throughout this paper, the nuclear-spin contribution $C_{\text {nuc }}$ has been subtracted from the data, assuming the form $C_{\text {nuc }}=\left(A_{0}+A_{1} H^{2}\right) / T^{2}$ with $A_{0}=7.58 \times 10^{-2} \mathrm{~mJ} \mathrm{~K} \mathrm{~mol}^{-1}$ and $A_{1}=$ $6.90 \times 10^{-2} \mathrm{~mJ} \mathrm{~K} \mathrm{~mol}^{-1} \mathrm{~T}^{-2}$ [5]. $C(H)$ of $\mathrm{CeCoIn}_{5}$ in the superconducting mixed state is quite unusual. This behavior is very different from the one expected for ordinary $s$ wave superconductors in which $C(H)$ linearly increases with $H$ due to the contribution of QPs trapped in the vortex cores. In many of anisotropic superconductors, a power law dependence of $C(H) \propto H^{\beta}$ with $\beta \sim 0.5$ has been observed and is attributed to the nodal QP excitations. The negative curvature of $C(H)$ in low fields seen in figure is thus consistent with the existence of gap nodes in $\mathrm{CeCoIn}_{5}$. However, the curvature of $C(H)$ changes sign above $2 \mathrm{~T}$ and becomes strongly positive at higher fields, as shown in the inset. Similar behavior is previously reported for $\boldsymbol{H} \|$ [001] [5], as well as in $\mathrm{Sr}_{2} \mathrm{RuO}_{4}$ for $\boldsymbol{H} \|$ [110] [21]. Although the reason for this field dependence of the specific heat in $\mathrm{CeCoIn}_{5}$ is unclear at the moment, it might be related to nonFermi liquid behavior near the quantum critical point. With increasing $H$ in the $c$-plane, we observed a small but discernible anisotropy in $C(H)$ as shown in figure 1 $C(\boldsymbol{H} \|[100])<C(\boldsymbol{H} \|[110])$.

The in-plane anisotropy of $C(H)$ can be demonstrated more clearly by measuring its field-angular dependence, and some of the results are shown in figure 2 First of all, we examined the contribution of the addenda (the lower trace of figure 2(a)),

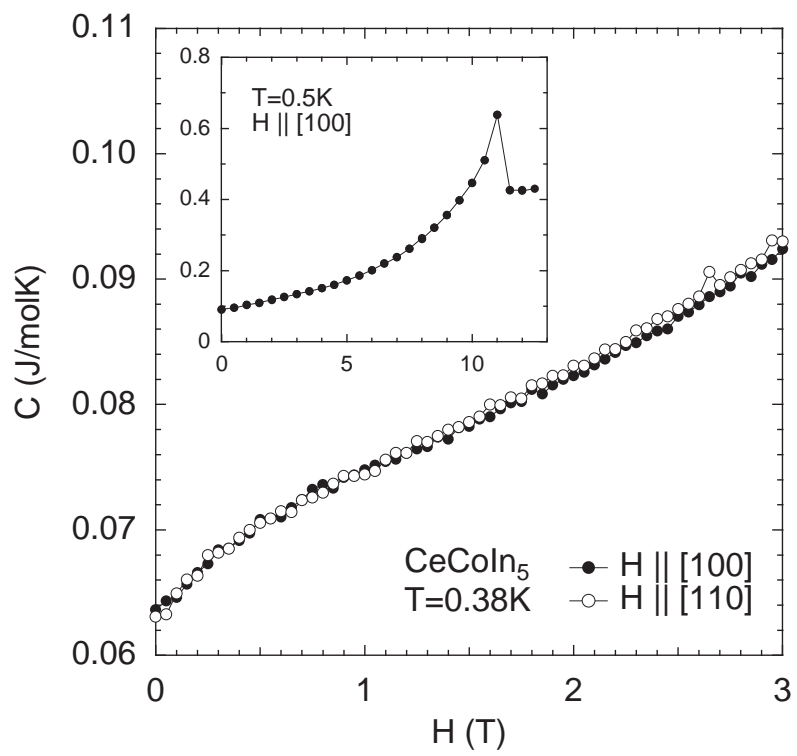

Figure 1. Field dependence of the specific heat $C(H)$ of $\mathrm{CeCoIn}_{5}$ for $\boldsymbol{H} \|[100]$ (solid circles) and [110] (open circles), measured at $T=0.38 \mathrm{~K}$. Inset: Overall field variation of $C(H)$ up to $12.5 \mathrm{~T}$ for $\boldsymbol{H} \|$ [100], measured at $0.5 \mathrm{~K}$. 


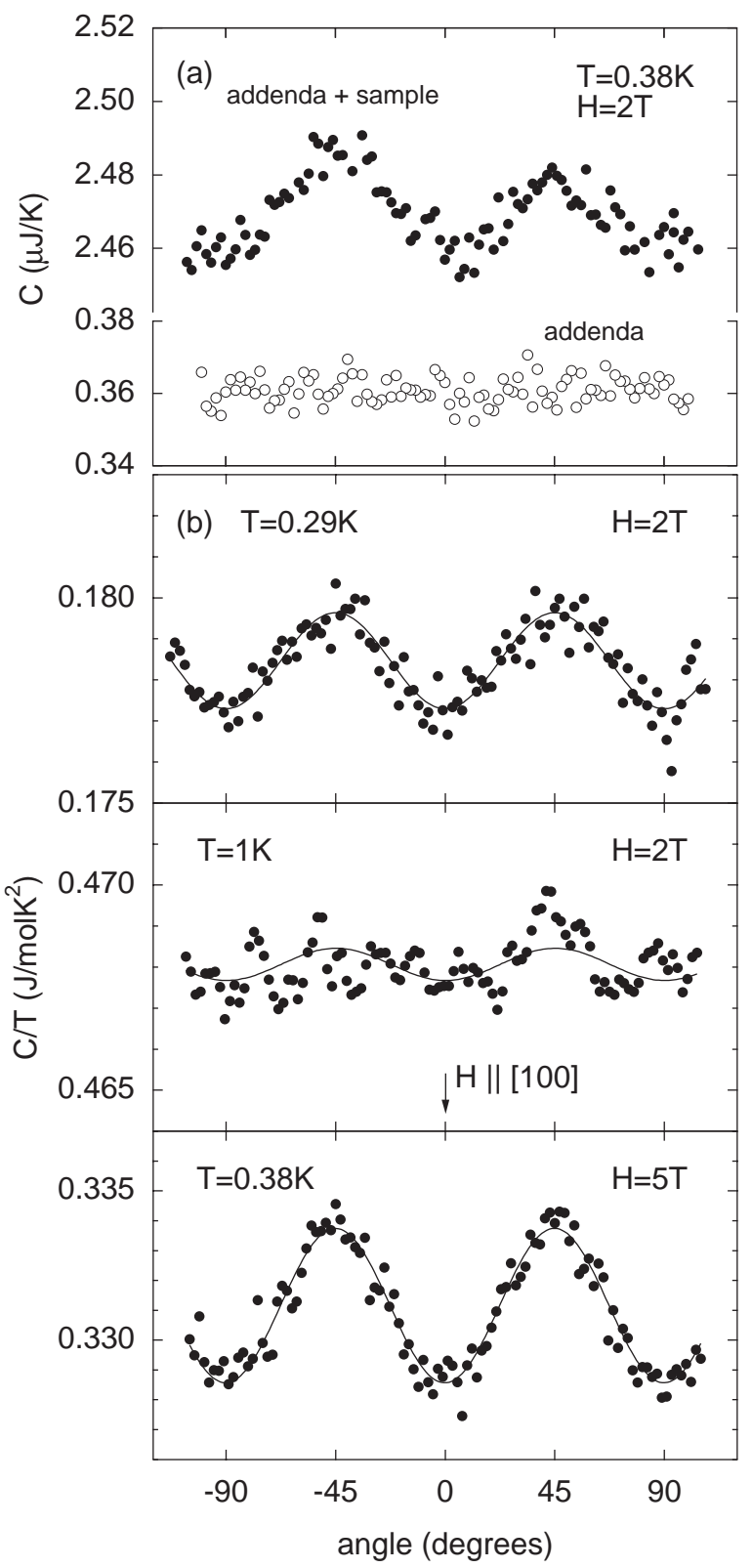

Figure 2. (a) Angular dependence of the total specific heat of $\mathrm{CeCoIn}_{5}$ (solid circles) mounted on the platform, in a field of $2 \mathrm{~T}$ rotated in the $c$-plane at $T=0.38 \mathrm{~K} . \theta=0$ is the [100] direction. Open circles are the results without the sample. (b) Field-orientational dependence of the specific heat of $\mathrm{CeCoIn}_{5}$ for $H=2 \mathrm{~T}$ measured at $T=0.29 \mathrm{~K}$ and $1 \mathrm{~K}$, and for $H=5 \mathrm{~T}$ measured at $T=0.38 \mathrm{~K}$. Solid lines are the fit to $C(H, \theta) / T=\left(C_{0}+C_{H}\left(1+A_{4} \cos 4 \theta\right)\right) / T$, where $C_{0}=0.0361,0.0631$ and $0.4042 \mathrm{~J} \mathrm{~mol}^{-1} \mathrm{~K}^{-1}$ for $T=0.29,0.38$ and $1 \mathrm{~K}$, and $A_{4}=-0.0217(2 \mathrm{~T}, 0.29 \mathrm{~K}),-0.0157(5 \mathrm{~T}, 0.38 \mathrm{~K})$ and $-0.0061(2 \mathrm{~T}, 1 \mathrm{~K})$. 
which is composed of the quartz platform, a thermometer $\left(\mathrm{RuO}_{2}\right.$ chip resistor $)$ and a heater. As can be seen in the figure, there is virtually no angular dependence in the addenda specific heat, implying that the field-orientational dependence of the thermometer is negligible. By contrast, there is a small but distinct fourfold oscillation in the raw specific heat data with the sample on the platform (the upper trace of figure 2(a)). Comparing these two data, it is obvious that the observed angular oscillation is intrinsic to the sample. No appreciable twofold component is observed in the oscillation, indicating that the magnetic field is well oriented along the $c$-plane.

The specific heat $C(H, \theta) / T$ of $\mathrm{CeCoIn}_{5}$ is shown in figure 2(b) for $H=2 \mathrm{~T}$ ( $T=$ 0.29 and $1 \mathrm{~K})$ and $5 \mathrm{~T}(T=0.38 \mathrm{~K})$ as a function of the field angle $\theta$ measured with respect to the $a$-axis. The fourfold oscillation is clearly seen at the base temperature of $0.29 \mathrm{~K}$ but rapidly fades away at higher temperatures. $C(H, \theta)$ can be decomposed into a constant and field-angle-dependent terms: $C(H, \theta)=C_{0}+C_{H}(1+A(\theta))$. $C_{0}$ is the zero-field term mainly due to thermally excited quasiparticles and phonons, whereas $C_{H}$ and $A(\theta)$ are field-dependent. The solid lines in figure 2(b) are the fitting results assuming a simple form $A(\theta)=A_{4} \cos 4 \theta$, by which we evaluated the amplitude of the fourfold angular oscillation. The sign of $A_{4}$ is negative, with the minima of $C(H, \theta)$ occurring along the $a$-directions. In figure 3 we plotted the field dependence of the relative amplitude $\left|A_{4}\right|$ as a function of $H / H_{c 2}$. Most remarkably, $\left|A_{4}\right|$ decreases monotonously with $H$ within the range of fields examined. The inset of figure 3 shows the temperature variation of the absolute amplitude $\left|A_{4}\right| C_{H} / T$ measured at $H=2 \mathrm{~T}\left(H / H_{c 2}=0.175\right)$. The fourfold angular oscillation in $C(H, \theta) / T$ rapidly diminishes with increasing $T$ and vanishes above $T_{c}$, implying that superconductivity

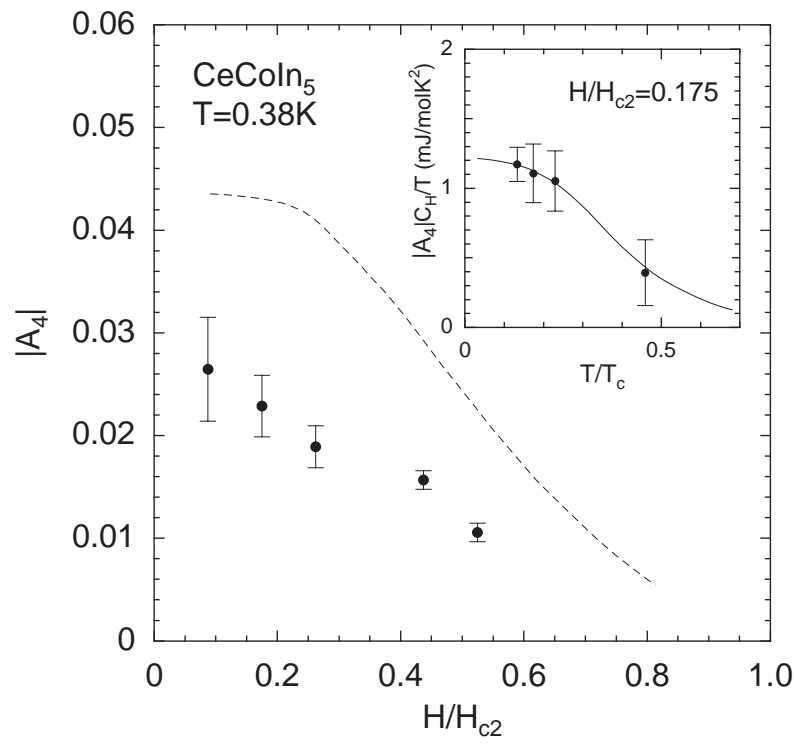

Figure 3. Field dependence of the amplitude $\left|A_{4}\right|$ of the fourfold oscillation at $T=0.38 \mathrm{~K}$, plotted as a function of reduced field $H / H_{c 2}$. The broken line is the calculated field variation for $\left|A_{4}\right|$ at $T=0$ assuming the $d_{x y}$-type gap node. Inset: temperature dependence of the absolute amplitude $\left|A_{4}\right| C_{H} / T$ measured at $H / H_{c 2}=0.175$. The solid line is a guide for the eyes. 
is responsible for its occurrence.

We now discuss the origin of the fourfold oscillation in $C(H, \theta)$. First of all, we should pay attention to the in-plane anisotropy of $H_{c 2}$, which is determined both by the gap topology and Fermi surface anisotropy; $H_{c 2} \|[100]$ is about $2.7 \%$ larger than $H_{c 2} \|$ [110] [19. The in-plane anisotropy of $H_{c 2}$ alone would also give rise to the angular dependence of ZEDOS. This is because the gap amplitude, which is a decreasing function of the reduced field $H / H_{c 2}$, becomes field-angle dependent under fixed $H$. This effect certainly becomes important when $H$ is near $H_{c 2}$, and enhances $C(H, \theta)$ for $\boldsymbol{H} \|[110]$ along which the system is closest to the normal state; the sign of the fourfold amplitude is the same as what we observed. However, in the limit of low fields $H \ll H_{c 2}$ the effect of the upper critical field can be disregarded. A crude estimate, assuming that $C(H)$ in figure 1 can be scaled linearly with $H_{c 2}(\theta)$, shows that the $\sim 3 \%$ anisotropy in $H_{c 2}$ would give the fourfold term $A_{4}$ of the order of $1 \%$ at $2 \mathrm{~T}$. This definitely overestimates the effect of $H_{c 2}$ anisotropy, but is still smaller than the observed one. Moreover, one expects the effect of the upper critical field to proliferate with increasing field. Instead, the oscillation amplitude is a decreasing function of $H$ up to at least $H \sim 0.5 H_{c 2}$. The strong temperature variation of the fourfold amplitude of $C(H) / T$ shown in the inset of the figure 3 is also hardly explained by the $H_{c 2}$ inplane anisotropy effect, because the upturn in $C(H) / T$ does not change much in these temperature regions [5]. Since the effect of $H_{c 2}$ anisotropy is incompatible with the data, we are led to conclude that the observed angular oscillation in $C(H, \theta)$ originates from the nodal structure, which has fourfold symmetry in the $a b$-plane.

In the mixed state of anisotropic superconductors enhanced contribution to ZEDOS is coming from the QPs with momentum along the gap node direction. The enhancement depends on the relative position of the gap and magnetic field direction. Accordingly, ZEDOS becomes field-angle dependent, and exhibits a characteristic oscillation with respect to the angle between $H$ and gap-node directions. This ZEDOS oscillation is directly reflected in the field-orientational dependence of the specific heat at low $T$, which takes minima (maxima) for $H$ parallel to the nodal (antinodal) direction [10, 12, 14. The clear minima of $C(H, \theta)$ along [100], which persist down to low fields, indicate that there are gap nodes along these directions. Here we performed the microscopic calculation of the field-angle dependent ZEDOS of clean $d$-wave superconductor with spherical Fermi surface. We solved numerically and self-consistently the quasi-classical Eilenberger equations. This formalism is a good approximation as long as $k_{F} \xi \gg 1\left(k_{F}\right.$ being the Fermi wave number and $\xi$ the coherence length), the condition which is met in $\mathrm{CeCoIn}_{5}$. It takes into account both the Doppler shift effect and the vortex core contribution on equal footing and without any further approximation. The result is presented with the broken line in figure 3 Although the predicted curve gives slightly larger amplitude than the experimental one, it explains the overall field dependence of our experimental result reasonably well. The discrepancy between the predicted and observed amplitude may partly arise from the fact that the measurements have been done at finite $T\left(T / T_{c} \sim 0.17\right)$, as inferred from the strong temperature variation of the amplitude predicted in Ref. [12]. The actual amplitude however gradually levels off below $0.5 \mathrm{~K}$. Impurities, which are always present, may reduce the oscillation amplitude at lower $T$. The present data thus strongly indicate that the gap symmetry of $\mathrm{CeCoIn}_{5}$ is most probably of $d_{x y}$ type.

The present results agree with the $\kappa(\theta)$ measurement on $\mathrm{CeCoIn}_{5}$ [17 in the point that the nodal structure has fourfold symmetry in the $c$-plane, but disagrees on the 
location of the nodes. $\kappa(\theta)$ shows a fourfold oscillation with the minima along the [010] and [100] directions, which is the same oscillation behavior as our $C(\theta)$ data. The authors of Ref. [17 assumed that this angular dependence of $\kappa(\theta)$ is dominated by the QP scattering time, which becomes largest (smallest) when the magnetic field is along the nodal (antinodal) directions, just opposite to the ZEDOS contribution. It is considered that the QP scattering effect is important at high temperatures, but the ZEDOS contribution becomes predominant with decreasing $T$. If the angular oscillation of $\kappa(\theta)$ had come from the QP scattering term, then its amplitude should change sign on cooling. The observed amplitude of the fourfold term in $\kappa(\theta)$ however continues to increase down to the lowest $T$ of $0.35 \mathrm{~K}$ [17. Whether the amplitude changes sign at still lower temperatures or not would be an interesting issue, but our $C(\theta)$ data implies that the ZEDOS effect is already predominant in $\kappa(\theta)$ at temperatures above $0.35 \mathrm{~K}$.

Present data also does not contradict the neutron scattering experiment by Eskildsen et al [9], who find the square vortex lattice oriented along the [110] direction. The result of neutron scattering experiment was argued to support the picture of $\mathrm{CeCoIn}_{5}$ as being the $d_{x^{2}-y^{2}}$ superconductor. However, the structure and the orientation of the vortex lattice is determined by the combined effect of the Fermi surface anisotropy and the gap structure. Which effect will prevail is a subtle question and the answer depends on many factors: field value, temperature, degree of Fermi surface anisotropy etc. Thus, the orientation of the square vortex lattice in tetragonal crystals may not serve as a conclusive test for the positions of gap nodes. Model calculation by Nakai et al 22] shows a variety of stable vortex lattice structures in tetragonal $d_{x y}$-wave superconductors. It is also shown that the low field square vortex lattice is oriented along [110] direction which is consistent with the neutron scattering experiment. It is interesting to point out that the square vortex lattice orientation should rotate by $\pi / 4$ in high fields if the $d_{x y}$ is indeed correct identification of the gap function.

In summary, we have performed the angle-resolved specific heat measurements on the heavy-fermion superconductor $\mathrm{CeCoIn}_{5}$ in a magnetic field rotating in the basal $a b$-plane. We observed a clear fourfold symmetry in $C(H, \theta)$ with the minima oriented along [100] directions, which comes from the field-angular oscillation of the zero-energy density of nodal quasiparticles. The results imply that the superconducting gap node of $\mathrm{CeCoIn}_{5}$ is located most likely along the [100] and [010] directions, suggesting the symmetry to be of $d_{x y}$ type.

\section{Acknowledgments}

We thank Y Matsuda, T Tayama and K Izawa for stimulating discussions. We are also grateful to Z Hiroi for his cooperation in specific heat measurements by PPMS. This work has partly been supported by the Grant-in-Aid for Scientific Research of Japan Society for the Promotion of Science.

\section{References}

[1] Hegger H et al 2000 Phys. Rev. Lett. 844986

[2] Petrovic C et al 2001 Europhys. Lett. 53354

[3] Petrovic C et al 2001 J. Phys.: Condens. Matter 13 L337

[4] Movshovich R et al 2001 Phys. Rev. Lett. 865152

[5] Ikeda S et al 2001 J. Phys. Soc. Japan 702248 
[6] Kohori Y et al 2001 Phys. Rev. B 64134526

[7] Curro N J et al 2001 Phys. Rev. B 64180514

[8] Chia E E M et al 2003 Phys. Rev. B 67014527

[9] Eskildsen M R et al 2003 Phys. Rev. Lett. 90187001

[10] Vekhter I, Hirschfeld P J, Carbotte J P and Nicol E J 1999 Phys. Rev. B 59 R9023

[11] Maki K, Yang G and Won H 2000 Physica C 341-348 1647

[12] Won H and Maki K 2001 Europhys. Lett. 56729

[13] Volovik G E 1993 JETP Lett. 58469

[14] Miranović P, Nakai N, Ichioka M and Machida K 2003 Phys. Rev. B 68052501

[15] Yu F et al 1995 Phys. Rev. Lett. 745136

[16] Izawa K et al 2001 Phys. Rev. Lett. 862653

Izawa K et al 2002 Phys. Rev. Lett. 89137006

[17] Izawa K et al 2001 Phys. Rev. Lett. 87057002

[18] Park T et al 2003 Phys. Rev. Lett. 90177001

[19] Settai R et al 2001 J. Phys.: Condens. Matter 13 L627

[20] Aoki $\mathrm{H}$ and Sakakibara $\mathrm{T}$ in preparation

[21] Nishizaki S, Maeno Y and Mao Z 2000 J. Phys. Soc. Japan 69572

[22] Nakai N, Miranović P, Ichioka M and Machida K 2002 Phys. Rev. Lett. 89237004 